Revista LIDER, 37(22), 2020, pp. 44-62

eISSN: $0719-526$

ISSN: 0717-0165

\title{
Hacia un paradigma latinoamericano crítico de los bienes comunes
}

\section{Towards a critical Latin American paradigm of common goods}

\author{
Daniel Montañez Pico \\ Universidad Nacional Autónoma de México, México. danielm9876@hotmail.com
}

\section{RESUMEN}

Los numerosos y pujantes estudios sobre los bienes comunes en América Latina suelen tener un marcado carácter capitalista y eurocéntrico. Frente a ello, diversas propuestas apuntan a comprender los problemas derivados de esas discusiones desde un punto de vista distinto, constituyendo las emergentes bases de un paradigma crítico a modo de descolonización de estos enfoques dominantes. En este trabajo se analizan algunos de estos esfuerzos provenientes de Bolivia y México, los cuales cuentan con una visión regional de conjunto para América Latina. Concluimos que aún queda camino en la construcción de un paradigma latinoamericano crítico de los bienes comunes y ofrecemos algunas ideas que puedan abonar a tan importante tarea.

Palabras clave: comunalidad; América Latina; descolonización; eurocentrismo

\section{ABSTRACT}

The numerous and powerful studies on the Common Goods in Latin America tend to have a marked Capitalist and Eurocentric character. Faced with this, various proposals aim to understand the problems arising from these discussions from a different point of view, constituting the emerging bases of a critical paradigm as a decolonization of these dominant approaches. This paper analyzes some of these efforts from Bolivia and Mexico, which have an overall regional vision for Latin America. We conclude that there is still a way to build a Latin American critical paradigm of the Common Goods and we offer some ideas that can contribute to this important task.

Key words: communality; Latin America; decolonization; eurocentrism

Recibido: 21 de julio de 2020. Aceptado: 19 de octubre de 2020. 


\section{Introducción}

La historia de la discusión moderna sobre los bienes comunes arranca aproximadamente en la Europa del siglo XVII. Ello no significa que anteriormente no hubiera sido temática de intenso debate en la región. Por ejemplo, el jurista Elio Marciano, de finales de la época "dorada" o "clásica" del Derecho Romano (siglos I-III), desarrolló la idea de "Res Communes Omnium" - cosas comunes a todos- en donde incluía el aire, el agua corriente, el mar y su litoral. El jurista extraía así una serie de recursos que solían estar en manos particulares o englobados dentro de lo que se conocía como "Res Publicae" - cosas públicas- hacia el ámbito de lo común, excediendo tanto el carácter privado como público de la propiedad y gestión de algunos recursos (Terrazas, 2012). La discusión continúo con entusiasmo en la Edad Media a través de las reflexiones sobre el Bien Común, intensamente desarrolladas, entre otros, por Tomás de Aquino en el siglo XIII. De este filósofo es muy conocida la sentencia jaculatoria que afirmaba "In extrema necessitate omnia sunt communia" -en extrema necesidad todo es comunidad-, con la que hacía referencia a la importancia de compartir en momentos de crisis aguda (Raffo, 1949). La expresión sería recuperada dos siglos más tarde por el predicador y filósofo alemán revolucionario Thomas Muntzer, quien lideró una gran rebelión campesina bajo el grito de "Omnia sunt communia" -todo es comunidad-, extendiendo a todo momento esa necesidad de compartir que Aquino solo veía necesaria en época de crisis. Hasta nuestros días este sigue siendo en Europa un grito muy recurrido en numerosos movimientos municipalistas en defensa de los bienes comunes, cuyo origen, curiosamente, venía de la frase de aquel filósofo medieval que creía, contrariamente a Muntzer y a estos movimientos, en la necesidad de una férrea jerarquía y desigualdad social.

Entonces, cuando decimos que la discusión moderna sobre los bienes comunes comienza en el siglo XVII, no estamos negando la existencia de estos y otros importantes antecedentes, sino ubicando el debate en los términos contemporáneos. Fue a partir del siglo XVII donde aparecen autores trabajando el tema en relación a la eficiencia en términos mercantiles de estos bienes. Una burguesía cada vez más influyente tomaba partido en la discusión e introducía desde sus intelectuales sus posiciones respecto al problema. Lo que hasta entonces había sido un debate más ligado a la subsistencia y a los conflictos territoriales entre el campesinado y la nobleza pasaba a ser mirado con la lupa del productivismo y el valor mercantil de cambio. En definitiva: no era sensato mantener los bienes comunes, ya que esa no era una forma efectiva de producir valor en términos de mercado y expandir la economía y el desarrollo capitalista. Un campeón de esta forma liberal de ver el mundo, John Locke, planteaba el problema en los siguientes términos: 
Dios ha dado a los hombres el mundo en común; pero como se lo dio para su beneficio y para que sacaran de él lo que más les conviniera para su vida, no podemos suponer que fuese la intención de Dios dejar que el mundo permaneciese siendo terreno comunal y sin cultivar. Ha dado el mundo para que el hombre trabajador racional lo use; y es el trabajo lo que da derecho a la propiedad, y no los delirios y la avaricia de los revoltosos y los pendencieros [...] Dios, al mandar que la tierra fuera sometida, estaba dando también la autoridad para apropiársela. Así que es la misma condición de la vida humana, la cual requiere trabajo y bienes materiales en los que trabajar, la que da lugar a que haya posesiones privadas (Locke, 2017 [1690], pp. 71/72-73)

Siguiendo esta visión de Locke, la mayoría se inclinaron a pensar desde esta manera de comprender el mundo que los bienes comunes tendrían que desaparecer en favor de la propiedad privada, que sería una forma más eficiente de gestionar la productividad del territorio. Sin embargo, hubo quienes también argumentaron lo contrario desde esa misma visión liberal del mundo. Es el caso de Hans Carl Von Carlowitz, un contemporáneo de Locke que trabajaba de administrador de minas en Alemania que se enfrentó al problema de la deforestación a causa de la actividad minera. Como la madera era indispensable para el desarrollo de esta industria y del país, la deforestación ponía en riesgo su existencia, por lo que defendió los bosques como parte de unos bienes comunes que habían de ser gestionados de forma sostenible. De hecho, se le considera un precursor del pensamiento sostenible al ser la primera persona que utilizó el término "sostenibilidad" en el sentido contemporáneo. En un tratado titulado "Silvicultura económica o sobre las instrucciones para la arboricultura salvaje” de 1713 lo exponía así:

Por lo tanto, el más grande arte, ciencia, industria e institución basada en ello [silvicultura] existe en esta región [Sajonia]. Como tal, se deben hacer esfuerzos para la conservación y el cultivo de la madera. Debemos aspirar a un uso continuo, resistente y sostenible, porque [los bosques] son algo indispensable, sin los cuales el país y sus forjas no podrían existir (Citado en Caradonna, 2014, p. 35, traducción propia)

La discusión sobre si los bienes comunes eran eficientes o no, sobre si había que suprimirlos, alentarlos o transformarlos, continúo acaloradamente hasta nuestros días. Pero no sería hasta la publicación de un polémico artículo de Garret Hardin que el debate entraría con fuerza en los círculos académicos contemporáneos. En "La tragedia de los comunes" Hardin defendió de forma muy seductora a través del uso del dilema del enemigo la ineficacia de mantener los tradicionales sistemas de gestión comunal de los recursos. Desde un punto de vista malthusiano abogó por la gestión privada de estos bienes ante el riesgo de inminente colapso natural del mundo (Hardin, 1968). Y la respuesta no tardó en llegar. La 
economista Elinor Ostrom capitaneó un proyecto de investigación internacional para demostrar lo contrario. En "El gobierno de los comunes" expuso de forma pormenorizada el trabajo de décadas de investigación sobre variados estudios de caso de gestión comunal de diferentes recursos a lo largo de todo el mundo demostrando que en muchos casos era una estrategia más eficiente y sostenible que la gestión pública o privada (Ostrom, 1990). La investigación le hizo merecedora del premio Nobel de economía en 2009 y el impacto de la obra en las ciencias sociales contemporáneas fue muy notorio, provocando una oleada de estudios sobre la gestión comunal de recursos naturales bajo su perspectiva teórica y metodológica que continúa hasta nuestros días.

Sin embargo, el enfoque institucional de Ostrom no terminó de convencer a quienes sostenían tesis críticas sobre el sistema económico capitalista. Bajo este punto de vista los trabajos de esta autora no ponían en tela de juicio la estructura de la explotación, incluyendo la gestión comunal de los bienes dentro del modo de producción capitalista. Además, sus esquemas, aunque utilizaban estudios de caso de todo el mundo, no atendían la diferencia estructural entre economías de "centro" y "periferia". En este sentido, epistemológica y metodológicamente su enfoque era claramente eurocentrista, cuestión que terminó deslizándose inevitablemente en la mayoría de estudios que toman su modelo para el análisis de casos en regiones "periféricas". Ante esta situación han emergido propuestas teóricas y metodológicas desde América Latina que proponen el análisis de los bienes comunes desde un horizonte epistemológico propio, dialogando con las propuestas críticas sobre los bienes comunes generadas en Occidente. A continuación analizaremos primero las bases del paradigma crítico occidental sobre los bienes comunes y su mirada sobre nuestra región latinoamericana, para después detenernos en las bases emergentes de un paradigma latinoamericano propio y terminar concluyendo con una serie de propuestas a modo de aporte para la construcción de dicho paradigma.

\section{El enfoque crítico occidental y su mirada sobre América Latina}

El primer teórico moderno occidental que abordó el problema de los bienes comunes desde una perspectiva crítica fue sin lugar a dudas Karl Marx. Desde sus más tempranos escritos muestra una conciencia ecológica frente a la depredación natural y social que suponía el sistema capitalista industrial liderado por la burguesía que en su época ya imponía un nuevo orden mundial y que dedicó toda su vida a estudiar y desentrañar. De su primera época destaca notablemente su escrito titulado "En defensa de los ladrones de leña" publicado en la Gaceta Renana en 1842 donde analiza el problema de la ilegalización de recogida de leña caída. El trabajo 
concluye que se trata de una actividad tradicional de subsistencia de las clases populares que no puede ser criminalizada solo por el interés económico de los grandes propietarios de tierra, que buscan enriquecerse todavía más a través de cobrar por la recogida de esos recursos que siempre habían sido de acceso y uso común. Para Marx la madera caída, muerta, no podía ser considerada propiedad particular en ningún caso, solo los árboles vivos podrían llegar a considerarse propiedad de alguien, por lo que la criminalización de esta actividad se mostraba jurídicamente insostenible. Además, estos llamados "ladrones" de leña eran en realidad la población trabajadora que utilizaba ancestralmente estos recursos para construir y calentar sus casas o cocinar, para subsistir, por lo que tampoco éticamente les podría ser negada esta actividad y menos bajo el argumento de enriquecer aún más a quienes ya son ricos (Marx, 2015 [1842]).

Aunque argumentos de este estilo ya habían sido esgrimidos por otros. Jean-Jacques Rousseau no solo había incendiado el corazón de Marx con sus proclamas por la voluntad y el interés general, poniendo énfasis en proteger a las clases populares menos privilegiadas. Era necesaria una profundización teórica mayor del problema para considerarle el primer gran teórico crítico de los bienes comunes. Será décadas más tarde cuando conseguiría adquirir ese estatus a través de las reflexiones contenidas en el capítulo XXIV del primer tomo de su magna obra, El Capital (1867). "La llamada acumulación originaria" es el penúltimo de los capítulos que componen su famoso estudio sobre economía política. Se trata de un estudio jurídico y social sobre la construcción histórica de las condiciones necesarias para impulsar el sistema capitalista, atendiendo especialmente al caso de Inglaterra. Después de estudiar el sistema de explotación desde el análisis de la mercancía, la generación de valor, plusvalía y el ciclo de acumulación de Capital, era necesario un colofón final que explicara los acontecimientos que habían posibilitado la existencia de dicho sistema. En este sentido rastrea, al menos desde el siglo XIII, toda una serie de políticas públicas y privadas destinadas al despojo y cercamiento de los bienes comunes, aquellos bienes tradicionalmente dedicados a la subsistencia comunitaria como los bosques, manantiales o litorales marítimos. Actividades relacionadas con la alimentación cotidiana o la salud comunitaria derivadas del uso común de estos bienes serían poco a poco privatizadas y reguladas en beneficio de los propietarios, quienes las convertirían de diversos modos en lucrativos negocios. La novedad que introduce Marx en este texto no es tanto la narración de los hechos, labor que ya había realizado anteriormente, sino su vinculación directa con la construcción histórica de un sistema político, económico y social, el capitalismo, destinado a explotar a la mayoría de las personas en el mundo. El despojo y cercamiento de los bienes comunes pasaban a ser un problema estructural que iba mucho más allá de la simple afrenta coyuntural a las clases populares, sino que era una de las formas de 
generar fuerza de trabajo "libre", se trataba de una de las principales estrategias para crear sujetos despojados de toda posibilidad de supervivencia, obligados así a vender su fuerza de trabajo al Capital para subsistir (Marx, 1998 [1867], pp. 221-245).

Y a continuación aparece por fin América. En un último y breve capítulo de $E l$ Capital, "La moderna teoría de la colonización", la conquista y despojo del continente aparecen como parte sustancial de esas estrategias de "acumulación originaria” de Capital. Pero el desarrollo de la cuestión es limitado y no atiende a las estrategias concretas propias de despojo de los bienes comunes en el continente. Para Marx la cuestión de la colonización era de suma importancia y tenía el plan de desarrollarla de forma pormenorizada, pero no le dio el tiempo, dejándonos solo, a demás de este capítulo, una serie de interesantes reflexiones desperdigadas en diferentes y pequeños textos (Marx y Engels, 1978).

Como sabemos, Marx tuvo un impacto notable en el pensamiento y la política del mundo moderno. Sin embargo, sus reflexiones críticas sobre los bienes comunes solo fueron atendidas y profundizadas en las últimas décadas a raíz del nuevo ciclo global de acumulación de Capital conocido comúnmente como neoliberalismo, en el que se han acentuado de nuevo las estrategias de despojo y cercamiento de los bienes comunes. A diferencia del enfoque de Hardin y Ostrom, que emergía ante la preocupación sobre el problema del cambio climático y la sostenibilidad generadas ante la industrialización masiva del mundo, estas reflexiones reaccionaban ante la renovada violencia estructural del Capital contra las clases populares. Para estos enfoques críticos se trataba de defender los bienes comunes y su gestión comunitaria no solo porque fueran formas más eficientes y sustentables de producir mercancías, sino porque su despojo implicaba la pérdida de diversas culturas y tradiciones ancestrales de subsistencia y, al fin y al cabo, de la vida misma. Se trataba no tanto de hacer comunitario el desarrollo, como proponían el enfoque liberal de Ostrom, sino de frenarlo, destruirlo y construir otro sistema social menos depredador del mundo y el ser humano. Esta serie de reflexiones críticas, que comenzaron tímidamente en los años 60 y se fueron haciendo más habituales desde los 70, fueron recogidas en espacios editoriales como The Commoner, revista que contó con 15 números entre el año 2001 y 2012 en donde se recuperó e incentivó el enfoque crítico sobre los bienes comunes de la mano de autores y autoras como Silvia Federici, George Caffentzis, Massimo de Angelis y el Midnight Note Collective entre otros.

En este resurgir del enfoque crítico sobre los bienes comunes, el análisis del caso latinoamericano fue crucial. La emergencia de movimientos indígenas en defensa del territorio desde los años 70 impactó profundamente en estos enfoques que atendieron especialmente los problemas del continente. Mencionaremos brevemente a tres de ellos para ilustrar la cuestión. 
En primer lugar, Ivan Illich. Pensador de origen austríaco que radicó durante muchos años en México, produjo una inspiradora y polifacética obra de crítica a la modernidad como civilización abordando cuestiones relacionadas con la crítica a sus modelos de educación, salud o desarrollo económico. Además, defendió las formas comunitarias de vida de los pueblos indígenas y acompañó algunas de sus demandas de defensa del territorio en México. Desde esta experiencia ofreció una famosa conferencia en Japón en 1982 titulada "El silencio es un bien comunal" en donde relacionaba el problema histórico del despojo de los bienes comunes con los que enfrentaban los pueblos americanos en los tiempos contemporáneos y el nexo que todo ello tenía con los problemas estructurales que habitan en la civilización moderna capitalista en la que nos encontramos. Por la claridad, contundencia y lucidez del texto nos permitimos reproducir un largo pasaje en donde expone su posición al respecto de esta discusión:

Distingamos al medio ambiente como bien común del medio ambiente como riqueza [...] Debemos distinguir entre los bienes comunales en los que se enmarcan las actividades para la subsistencia de la gente, y las riquezas de la tierra (los recursos naturales) que sirven para la producción económica de aquellos bienes de consumo sobre las que se asienta la vida actual [...] Antes, en cualquier sistema jurídico, la mayor parte del entorno se consideraba como bien comunal, con el que la mayoría de la gente podía abastecer sus necesidades básicas sin tener que recurrir al mercado. Después del cercamiento, el entorno natural se tornó principalmente una riqueza al servicio de "empresas" que, al organizar el trabajo asalariado, transformaron la naturaleza en bienes y servicios de los que depende la satisfacción de las necesidades de los consumidores [...] La apropiación de los campos de pastoreo por parte de los señores fue desafiada, pero la más fundamental transformación de esas áreas (y de las calles) de bienes comunales a recursos, aconteció - hasta hace muy poco- sin ser objeto de crítica. La apropiación del entorno por la minoría fue claramente reconocida como un abuso intolerable. Pero la aún más degradante transformación de las personas en miembros de una fuerza de trabajo industrial y consumidores fue tomada -hasta hace pococomo algo natural [...] Este cambio de actitudes puede ilustrarse mejor si pensamos en las calles en vez de considerar las áreas de pastoreo. Qué enorme diferencia vemos en los barrios de la ciudad de México durante los últimos veinte años. Entonces las calles de los barrios eran realmente bienes comunales. Alguna gente las utilizaba para vender hortalizas y carbón de leña. Otros colocaban sus sillas en las aceras para beber café o tequila. Otros se reunían en la calle para decidir quién sería el nuevo representante del vecindario, o para determinar el precio de un asno. Otros conducían sus asnos por entre la multitud, caminando próximos a sus bestias de carga; otros montaban en sus sillas. Los niños jugaban en las zanjas y, aún así, los 
caminantes podían usar la calle para ir de un sitio a otro. Las calles no fueron construidas por la gente. Como cualquier otro bien común, la calle misma era el resultado de la gente que allí vivía y tornaba habitable ese espacio. Las viviendas que franqueaban las calles no eran hogares privados en el sentido moderno: garajes para el depósito nocturno de los trabajadores. El umbral separaba aún dos espacios vivientes, uno íntimo y otro común. Pero ni los hogares en su sentido íntimo ni las calles como bienes comunales sobrevivieron al crecimiento económico. En los nuevos barrios de la ciudad de México las calles no son ya para la gente. Son ahora carreteras para coches, para autobuses, taxis y camiones. La gente es difícilmente tolerada en las calles a menos que se dirija hacia la parada del autobús. Si ahora la gente se sentara o detuviera en las calles sería un obstáculo para el tránsito, y el tránsito sería peligroso para quien así lo hiciere. La calle fue degradada, de bien comunitario a un simple recurso para la circulación de vehículos. La gente ya no puede circular por sus espacios, el tránsito desplaza su movilidad. Sólo puede circular cuando se le acota y se le traslada (Illich, 2007 [1982], pp. 3-5)

En segundo lugar, Silvia Federici. Pensadora italiana cofundadora de The Commoner que milita desde hace décadas en movimientos feministas y ha tenida la posibilidad de conocer de cerca el despojo de bienes comunes en regiones de África y América Latina en las que ha trabajado y colaborado intensamente. Fruto de su experiencia elaboró la monumental obra Calibán y la bruja: mujeres, cuerpo y acumulación originaria en la que, desde un punto de vista marxista, analizaba el papel de las mujeres dentro de los procesos de despojo de los bienes comunes que posibilitaron el nacimiento del capitalismo como sistema mundial. La autora concluye que las mujeres sufrieron específicamente en este proceso debido a la histórica cercanía que mantenían con estos bienes, de donde extraían conocimientos medicinales entre otras cosas. Es por ello que en los procesos de su defensa fueron las primeras en reaccionar y liderar las diferentes luchas, razón por la que comenzaron a criminalizarlas y reprimirlas para disciplinarlas dentro de las nuevas concepciones del patriarcado capitalista. Es entonces cuando surgió la llamada caza de brujas, un dispositivo represivo organizado para deshacerse de las mujeres que no querían adaptarse a los cambios del sistema y acabar con su liderazgo dentro de los procesos de defensa de los bienes comunes. Federici plantea que esta caza de brujas continúa hasta nuestros días y que el neoliberalismo la ha recrudecido, sufriendo de nuevo específicamente mucho más las mujeres en los procesos de despojo, asistiendo a renovados feminicidios sistémicos. La obra incluye un capítulo sobre la caza de brujas en América Latina y trata de relacionar la caza de brujas con la colonización del continente, exponiendo ambos acontecimientos como aquellos dos grandes procesos genocidas que parieron el sistema capitalista -de ahí la referencia en el título de la obra al personaje shakesperiano de Caliban que retoma 
desde la reflexión crítica que hizo sobre él el maestro cubano Roberto Fernández Retamar- (Federici, 2013). Además, la autora ha argumentado junto a George Caffentzis sobre la necesidad de erigir un paradigma crítico integral y anticapitalista sobre los bienes comunes que haga frente a posturas como las de Ostrom, que tan solo consiguen maquillar un poco la violencia sistémica del capitalismo (Federici y Caffentzis, 2015).

Por último, el Comité invisible. Colectivo heredero de la revista francesa de crítica radical Tiqqun editada a finales de los años 90 que ha estado relacionado con experiencias de colectivización agraria en Francia. Desde 2004 han publicado varios libros en donde proponen caminos de acción política radical y revolucionaria desde el análisis de diferentes revueltas contemporáneas puestas en relación con experiencias de colectivización. En una de sus últimas obras, A nuestros amigos, incluyen un capítulo denominado "Omnia Sunt Communia" en el que repasan los sentidos de distintas manifestaciones contemporáneas como los Indignados de Europa, el movimiento Occupy de EEUU o la Primavera Árabe para incidir en la importancia de la figura de la comuna, la cual va mucho más allá del mero sentido mercantil que encuentran en la propuesta de gestión comunal de los recursos propuesta por Ostrom. Además, construyen sus ideas en un intenso diálogo con la propuesta de la "comunalidad" oaxaqueña de México, mostrando un interés y respeto por las formas de lucha y defensa de los bienes comunes propias de América Latina (Comité invisible, 2015).

Todos estos aportes son muy interesantes para comprender las continuidades existentes entre el comienzo del enfoque crítico sobre los bienes comunes del siglo XIX hasta sus manifestaciones más contemporáneas del siglo XXI. Pero a la hora de analizar el problema en América Latina no suelen atender aspectos de su propia historia del despojo de los bienes comunes en la región, adaptando la crítica de origen marxista basada en el análisis del proceso histórico europeo al caso latinoamericano. Es decir, son críticos pero se mantiene en mayor o menor medida un eurocentrismo epistemológico en sus acercamientos. Es necesario entonces atender a propuestas contemporáneas emergentes que están tratando de dilucidar la cuestión desde y para la región.

\section{Las bases de un emergente enfoque latinoamericano crítico}

Ante el avance contemporáneo del despojo de los bienes comunes en América Latina han emergido en la región una serie de enfoques teóricos y metodológicos relacionados con la temática que dialogan con el enfoque crítico occidental 
anteriormente expuesto. Nos detendremos específicamente en el análisis de propuestas surgidas en Bolivia, Venezuela y México.

\subsection{La forma y sistema comunal en el grupo Comuna, Bolivia}

A finales de los años 90 se conformó en Bolivia un espacio de intercambio y producción intelectual colectiva conocido como el grupo Comuna. Intelectuales, activistas y estudiantes de diversa procedencia, generalmente vinculados a diversos movimientos y organizaciones sociales del país, se fueron reuniendo periódicamente para compartir sus reflexiones en torno a las posibilidades de cambio social y político en Bolivia. Algunas de las figuras más conocidas y destacadas del grupo fueron Luis Tapia, Raúl Prada, Raquel Gutiérrez, Álvaro García Linera, Óscar Vega y Félix Patzi. El grupo trató de teorizar y acompañar el movimiento de intensa transformación que vivía el país y que dio lugar a la Guerra del Agua en el año 2000, la Guerra del Gas en el 2003 y, finalmente, el proceso constituyente de 2006 y los sucesivos gobiernos del Movimiento al Socialismo (MAS) presididos por Evo Morales (Kanahuaty, 2015). Una vez que el proceso de cambio se institucionalizó, el grupo Comuna fue disolviéndose y cada figura continuaría sus propios caminos de pensamiento y acción social, algunas vinculadas al gobierno -como el actual vicepresidente, Álvaro García Linera, el consejero de la Fundación Cultural del Banco Central de Bolivia, Óscar Vega, o el que llegó a ser candidato del MAS para el departamento de La Paz, Félix Patzi- y otras incluso confrontadas con el mismo por considerar que habían traicionado al llegar al poder el mandato de transformación del pueblo -como Raúl Prada o Raquel Gutiérrez.

El grupo Comuna estuvo intensamente influenciado por los movimientos indígenas que habían emergido desde los años 70 en el país. Seguramente sea Bolivia el territorio de América Latina donde se desarrolló una respuesta más contundente y radical frente al paradigma institucional paternalista del indigenismo, proponiéndose enfoques y políticas indianistas desde las propias comunidades que pretendían transformar todo el sistema social y no solo incluirse como minorías folclóricas en el mismo. Es por ello que la reflexión sobre la comunidad y lo comunal tomó en las discusiones de este grupo un papel central y fueron intensamente desarrolladas, enfatizando cuestiones relacionadas con la propiedad, economía y política comunal que podían constituir las bases de un cambio social anticapitalista. Revisaremos tres formulaciones emanadas de este contexto.

En primer lugar, Álvaro García Linera. Matemático y teórico político boliviano vinculado a diversas organizaciones políticas desde los años 80 como el Ejército Guerrillero Túpac Katari (EGTK), por cuya actividad acabó en prisión durante cinco años entre 1992 y 1997. En la cárcel elaboraría uno de sus más logrados libros, Forma valor y forma comunidad, que constituiría una influencia notable en el posterior 
grupo Comuna. La obra contrapone la visión marxista del valor dentro del sistema capitalista con la de la sociedad, economíay política emanada de sociedades no-capitalistas. Como Marx no desarrolló sistemáticamente esta última cuestión, Linera recupera todos sus aportes desperdigados, los sistematiza, y desde ahí avanza hacia una comprensión marxista de la cuestión comunitaria no-capitalista. Su intención era confrontar el linealismo histórico de la visión marxista ortodoxa y adentrarse en la comprensión estructural crítica de las formaciones sociales comunitarias no-capitalistas con dos objetivos. Primero, comprender aquellas formaciones sociales que aun eran mayoritarias en su país. Segundo, dilucidar cuáles de sus formas podían servir para construir un horizonte político no-capitalista contemporáneo. El autor concluye que la nueva política revolucionaria de Bolivia tendrá que estar marcada por la comprensión y vivencia comunitaria propia de los pueblos andinos de su región, pero teniendo que sublimar su nivel local hacia una formación más global que abarque la toma del poder institucional hegemónico en una especie de "comunalización del Estado" que denomina el "Ayllu universal", haciendo referencia a universalizar el Ayllu, aquella formación territorial comunitaria propia de los pueblos andinos de Bolivia. Así, para Linera eran igual de nocivos los enfoques marxistas ortodoxos que no tomaban en cuenta los aportes del mundo de lo comunal como aquellos posmodernos que veían las comunidades con un idealismo que paralizaba toda posibilidad de transformación de lo comunitario en un horizonte político más amplio (García Linera, 2009 [1995]).

En segundo lugar, Raquel Gutiérrez. Matemática y socióloga mexicana vinculada a diversos movimientos sociales que terminó recalando a finales de los años 80 en Bolivia vinculándose al EGTK y, más adelante después de una temporada en prisión, al grupo Comuna y diferentes movimientos sociales que provocaron la constituyente. En el año 2001 publicó un importante texto titulado "Forma comunal y forma liberal de la política" en donde contraponía la teoría política liberal basada en la ficción contractual de la delegación, que denomina "irresponsabilidad civil", con una emergente teoría política comunal basada en el control material de las condiciones de existencia por parte de las comunidades, que denomina "soberanía social”. El trabajo realiza una reconstrucción histórica de ambas tendencias y termina apostando por la comunal, la cual considera además confrontada inherentemente al sistema capitalista de producción de valor (Gutiérrez, 2001). El texto muestra sintonía argumental con la anterior propuesta analizada de la "forma comunidad" de García Linera, pero no da muestras de pretender sublimar lo comunitario hacia un horizonte universal más global como en aquel caso.

Por último, Félix Patzi. Sociólogo aymara paceño vinculado desde su juventud a movimientos indígenas de su región, desde 2006 entró a formar parte del gobierno del MAS liderado por Evo Morales como ministro de educación entre otros cargos y 
actualmente ejerce como gobernador de La Paz desde su propio movimiento denominado el "Tercer sistema". Este nombre proviene de sus tiempos como intelectual adherido al grupo Comuna y hace referencia al sistema comunal que tiene que ser distinguido del sistema liberal capitalista y del socialista. El desarrollo teórico de esta idea recuerda a la propuesta de Linera, pues también aboga por transformar y sublimar lo comunitario hacia un horizonte político de totalidad que tome el poder, pero su elaboración es menos crítica en términos marxistas. A diferencia de Linera, Patzi sí profundiza y elabora propuestas de cómo sería esa sublimación comunitaria, defendiendo ideas como la conformación de empresas comunitarias o la construcción de una democracia comunal. Su propuesta plantea descolonizar y comunalizar el Estado y la economía, pero sin preocuparse mucho del sistema de producción y distribución de mercancías propio del capitalismo global. Así que, en este sentido, su propuesta podría parecerse más a la "tercera vía" de Giddens, que por cierto cita ampliamente, pero bajo las coordenadas civilizatorias de los pueblos indígenas de Bolivia. De esta forma se trata de una propuesta que podría dialogar con la visión de Ostrom, pero elevando ese tipo de gestión local comunitaria de recursos a ámbitos nacionales e incluso internacionales en una especie de construcción de una "modernidad indígena" (Patzi, 2004).

\subsection{La comunalidad oaxaqueña, México}

Desde los años 70 emergieron en Oaxaca reflexiones en torno a lo que denominaron "comunalidad". La primera persona en utilizar y elaborar el concepto fue el antropólogo del pueblo mixe Floriberto Díaz, quien utilizaba el término para expresar "la esencia fenoménica que define la inmanencia de las comunidades indígenas" (Díaz, 2007). Paralelamente, otro antropólogo del pueblo zapoteco, Jaime Martínez Luna, comenzó a usar el término para referirse al "modo de vida de los pueblos indios de Oaxaca" (Martínez Luna, 2010). Ambos eran producto de un movimiento propio de la intelectualidad emergida desde las comunidades indígenas que reaccionaba ante el pensamiento y las políticas públicas del indigenismo, entendido este como aquella política paternalista, más o menos conservadora, dedicada a pensar cómo organizar e incluir a los pueblos indígenas dentro de la sociedades y estados nacionales. Cansados de escuchar cómo eran sus pueblos y qué debían hacer para desarrollarse, emergieron figuras como Floriberto y Jaime reclamando construir definiciones y teorías de las comunidades desde ellas mismas y sus propias realidades. Ese esfuerzo les llevó a realizar diversos esquemas, en donde destacaban como ejes de la comunalidad cuestiones como el territorio, la espiritualidad, las formas de gobierno y autoridad propia, la autonomía o la educación y el trabajo comunal. Los esquemas fueron evolucionando, hasta llegar a formulaciones recientes como las últimas de Jaime Luna que expresa la idea de 
comunalidad a través de la metáfora del trébol de cuatro hojas marcadas por: 1. El suelo que pisa la gente; 2. La gente que pisa ese suelo; 3. Lo que hace la gente que pisa ese suelo; y 4. Lo que consigue con su hacer la gente que pisa ese suelo (Martínez Luna, 2015, p. 105). El enfoque tuvo un impacto considerable en la región y en el país, teniendo numerosos seguidores y continuadores como Juan José Rendón, Benjamín Maldonado, Carlos Manzo o Arturo Guerrero.

Al ser al día de hoy ya toda una tradición intelectual con diversas ramificaciones, hay diferentes formas de entender, clasificar y definir la comunalidad. Pese a ello, en síntesis podríamos decir que uno de sus grandes aportes consiste en establecer modelos de análisis comunitario que propician el conocimiento de la comunidad desde las propias concepciones de la comunidad. Para ello, los autores tendrían que trascender las barreras disciplinarias que aprendieron en la universidad abriéndose a un abanico de conocimientos más amplios que exceden el ámbito académico y regresan al problema de la producción del conocimiento desde las colectividades mismas. Este ejercicio derivó en un rescate filosófico desde las propias lenguas y actividades cotidianas de cada comunidad que dio pie a la creación de este enfoque, desde donde se concluyó que el principio fundamental que organizaba a sus pueblos indígenas era la comunalidad, el sentido comunitario de existencia. Además, al tratarse de intelectuales "entre dos mundos", supieron, una vez construidas las primeras hipótesis sobre el ser comunitario indígena, ponerlas en diálogo con las filosofías de la sociedad occidental capitalista dominante, concluyendo que sus formas de vida comunales se diferenciaban y confrontaban con su paradigma social basado en el individualismo. En este sentido, las propuestas políticas de la comunalidad trascienden el horizonte autonomista de los indigenistas con la pretensión de llevar sus formas de vida y sus hallazgos sociales derivados al resto de la sociedad nacional, con el propósito de transformar el mundo entero en un sentido comunal. De esta forma se trata de un enfoque casi inverso al anteriormente estudiado de Venezuela, donde se parte de la realidad comunitaria para afectar desde ahí el resto de escalas sociales.

La única objeción, o más bien señalamiento a modo de diálogo con esta interesante corriente de pensamiento, sería considerar el lugar de estas "comunalidades" en el esquema de la economía política capitalista hegemónica, cuestión que no suele ser muy atendida por la corriente. El modelo colonial de poder que los hispanos impusieron desde el siglo XVI en la región se basó en una reorganización colonial de los modos comunales de vida indígenas mediante los llamados "repartimientos" en un nuevo modelo que denominaban "Repúblicas de indios". Este modelo organizaba a las poblaciones autóctonas en comunidades que tenían cierta autonomía para resolver sus asuntos internos y autogobernarse. Era necesario mantener espacios de reproducción de vidas indígenas que, además de 
pagar los consiguientes tributos en especias a la Corona, se mantuvieran como fábricas de mano de obra barata para trabajar en las minas y servir como ejércitos de reserva a la espera de tener que apoyar en las guerras de invasión del continente lideradas por los hispanos contra otros pueblos indígenas. Este sistema de "comunidades coloniales" fue atacado más adelante por gobiernos criollos de diversa índole y sigue siendo atacado por algunos sectores políticos que preferirían que no existieran pueblos indígenas. Sin embargo, su esencia se ha mantenido a través de la historia hasta nuestros días como demuestran numerosas comunidades indígenas adheridas a diferentes partidos políticos, los cuales entienden que se trata de un modelo funcional a la reproducción del capital. Aunque en este punto es muy importante señalar que también hubo muchas comunidades que se mantuvieron en resistencia y no dejaron que los hispanos designaran y educaran a sus líderes o les despojaran el territorio y sus recursos, desarrollando lo que podríamos llamar "comunalidades radicales" o de "ruptura" con el régimen capitalista y colonial, las cuales también persisten dignamente hasta nuestros días. De alguna manera se puede entender que muchos pueblos indígenas hayan entrado al juego del sistema colonial como forma de mantener sus sistemas comunales de vida en un contexto hostil en un ejercicio de "antropofagia cultural" como decía el modernista brasileño Oswald de Andrade. Pero comenzar a nombrar los problemas puede resultar un buen comienzo en el objetivo de transformar las relaciones sociales y patear el tablero político, ya que existe un fondo de comunalismo ancestral en todas estas comunidades, sean o no coloniales, del que hay muchísimo que aprender, como bien enseñan los trabajos de la corriente de la comunalidad oaxaqueña. Que esa energía sea conducida hacia la liberación y la transformación social y no hacia la reproducción del capitalismo y el colonialismo es uno de los principales desafíos políticos de la lucha social en América Latina. La diferenciación entre "comunalidades coloniales" y "comunalidades de ruptura", "radicales", o como se puedan denominar, podría ser de gran utilidad en esta tarea.

\subsection{La producción de lo común en la escuela de Puebla, México}

Por último, nos referiremos a la producción intelectual emanada desde el Posgrado de Sociología de la Benemérita Universidad Autónoma de Puebla en México. Desde este espacio, Raquel Gutiérrez, a quien ya vimos en la anterior experiencia del grupo Comuna en Bolivia, ha organizado en los últimos años un espacio horizontal de reflexión y producción colectiva de conocimiento que atiende a estudiar e impulsar lo que ha denominado "horizonte comunitario-popular". Este horizonte político se expresa desde experiencias de autoorganización comunitaria que exceden el ámbito público y privado organizando "islas de nuevos mundos adentro del mundo viejo", sin significar por ello que no dialoguen y se relacionen con 
experiencias que sí le apuestan al horizonte estatal siguiendo lo que denomina como "horizonte nacional-popular". Además, el apellido "popular" sirve para decantar hacia la izquierda la balanza y que no pueda ser confundido este horizonte con experiencias capitalistas de tipo comunitario como las defendidas por Ostrom en sus estudios (Gutiérrez, 2017).

El enfoque del horizonte comunitario-popular se asienta sobre el estudio de la "producción de lo común", que dialoga intensamente con el enfoque crítico sobre los bienes comunes elaborado en Occidente, especialmente con los aportes de Silvia Federici, por lo que, como sucede con ella, la dimensión feminista está absolutamente presente en el enfoque vertebrándolo de forma fundamental. Con "producción de lo común" se hace referencia a la existencia en numerosos movimientos y experiencias de colectivización de todo tipo de un modo de producción no-capitalista en el que prevalece el valor de uso sobre el valor de cambio, siguiendo en esta última reflexión las propuestas sobre la temática elaboradas por el economista ecuatoriano radicado en México Bolívar Echeverría. En este sentido, se hace hincapié en que se trata de modelos de comunalización de la vida imperfectos, no se trata de un enfoque idealista, sino de un paradigma procesual que atiende a experiencias de comunalización parciales dentro de un mundo sobredeterminado por la lógica liberal del capitalismo.

La propuesta ha tenido un número de seguidoras y seguidores considerables, entre los que destacan Mina Navarro, Lucía Linsalata o Huáscar Salazar. Así, desde el espacio de reflexión poblano han venido realizando numerosos aportes que estudian esta "producción de lo común" dentro de diversas experiencias de todo el continente latinoamericano e incluso en otras regiones del mundo, atendiendo a dimensiones de género, urbanas, rurales, políticas, laborales y un largo etc. de cuestiones (Navarro, 2016; Linsalata, 2015; Salazar, 2017). En todos estos trabajos se enfatiza que, a diferencia de como se suelen ver los bienes comunes en los enfoques clásicos, lo común no son cosas, sino relaciones sociales. Desde este enfoque se remarca que la importancia de que un bosque, lago, mar, litoral, fábrica, restaurante o panadería sea comunal no radica en la propiedad oficial del "recurso", sino sobre todo en las relaciones sociales comunales no-capitalistas que sostienen ese recurso y justamente por ello lo convierten en comunitario. Antes que poner la centralidad en el recurso y su eficiencia, se concentran en estudiar la sociedad que consigue producir y reproducir comunalmente el mismo. Es por ello que el enfoque trasciende en numerosos planos y dimensiones el objeto de estudio típico de las investigaciones sobre bienes comunes elaboradas desde los paradigmas clásicos, resituando el interés por las relaciones sociales mismas que los sostienen.

Aunque hemos de advertir que este señalamiento a las relaciones sociales que sostienen lo común, más allá de centrarse solo en lo que puedan producir o poseer 
comunalmente, es algo que ya había sido señalado en cierta medida por la tradición crítica de los bienes comunes europea analizada en el anterior epígrafe. Conceptos como "entramado comunal" u "horizonte comunitario-popular" vienen a enfatizar estos temas y a darle distintos matices pero no ofrecen grandes avances teóricos sobre la cuestión. En este sentido, es interesante la crítica a esta corriente vertida por el comunalista oaxaqueño Carlos Manzo, quien ha señalado en distintas intervenciones públicas que este concepto de "lo común" se fundamenta en la tradición crítica marxista de los "commons" y de alguna manera se inserta dentro de su genealogía histórica de crítica a los cercamientos de bienes comunes en la época medieval europea. Concordamos con este autor en que la escuela de Puebla no ofrece una genealogía histórica propia del problema del despojo de los bienes comunes en América Latina, realizando más bien una aplicación del modelo crítico europeo a diversos casos latinoamericanos. Quizás el aporte teórico más relevante de esta corriente sea introducir en el debate las ideas sobre la "preeminencia del valor de uso sobre el valor de cambio" de Bolívar Echeverría. Pero en ese ejercicio al final lo que se consigue es introducir dentro del concepto de "lo común" experiencias tan diversas como el cuidado comunal del agua por comunidades indígenas aymaras en Bolivia hasta la creación de huertos urbanos en Ciudad de México. Este es un criterio que homogeneiza demasiado las diferencias entre las distintas experiencias y sus genealogías históricas que, además, impide ver la realidad de la existencia de una dimensión comunal dentro de la reproducción de capital en América Latina, donde muchas veces se aprovechan y refuncionalizan lógicas comunales de los pueblos y experiencias en las que "predomina el valor de uso sobre el valor de cambio" para hacerlas eficientes al modelo de acumulación de capital global.

\section{Conclusiones}

Como se podrá comprobar, todos estos enfoques estudiados provenientes de Bolivia y México nos ofrecen excelentes miradores desde donde apreciar diferentes problemas sociales contemporáneos que atraviesa nuestro continente. Pero, además, sin desmerecer aspectos de los enfoques clásicos y críticos provenientes de Occidente, sostenemos que nos pueden servir de punto de partida para construir un necesario paradigma crítico, teórico y metodológico sobre los bienes comunes en América Latina. Para ello, a modo de síntesis conclusiva resaltaremos cuáles son los elementos que podríamos retomar de cada tradición en vías a la construcción de un paradigma crítico y descolonizador de los bienes comunes en América Latina.

Del grupo Comuna de Bolivia rescatamos el creativo diálogo producido entre las visiones comunitarias del indianismo con la tradición marxista heterodoxa. A modo de profundización de las tesis de Mariátegui, el grupo consiguió ofrecer una 
interpretación de las comunidades históricas campesinas indígenas de su región en clave política y económica que mostraban la potencia de lo comunitario para la transformación política de toda su sociedad, huyendo tanto del paternalismo indigenista como de las visiones marxistas ortodoxas linealistas de la historia, labor en la que, dicho sea de paso, fue fundamental la influencia de la obra de Fausto Reinaga y René Zavaleta.

Por otro lado, el caso de la comunalidad oaxaqueña expone un ejercicio excelente para apreciar la propia visión de las comunidades de nuestra región sobre sus sistemas comunales de existencia, más allá de los análisis antropológicos e indigenistas, desde sus propias formas de construir el conocimiento. Además, los esfuerzos provenientes del grupo de Puebla expanden la definición de los bienes comunes hacia el concepto de "lo común", para enfatizar que, desde un enfoque crítico, los bienes comunes son ante todo relaciones sociales comunales que los sostienen como tales y que, más que tratar de expandir los modelos comunales de propiedad, de lo que se trata es de expandir las formas de vida comunal en toda América Latina.

Los aportes de estos enfoques emergentes nos permitirán establecer brújulas propias para el análisis de lo comunal en América Latina, dimensión que es atacada desde diferentes frentes, tanto conservadores y liberales como progresistas, los cuales suelen compartir un fanatismo por proyectos extractivos y de reprimarización de la economía nacional, que ataca en muchos casos la autonomía territorial de los diversos ensayos sociales comunales de nuestra región. En definitiva, aún queda camino en la construcción de un paradigma crítico y descolonizador latinoamericano sobre los bienes comunes, tan importante para hacer frente a estos ataques cada vez más recrudecidos, pero contamos en nuestra región con experiencias, esfuerzos y bases teóricas emergentes tremendamente originales y valiosas desde donde avanzar en tan necesaria tarea.

\section{Agradecimientos}

Este texto es parte del proyecto docente "Bienes comunes, movimientos sociales y sostenibilidad en América Latina" impartido en el posgrado de Ciencias de la Sostenibilidad de la UNAM, México. Agradezco a Fernando González Lozada y Luis Castillo Farjat, coprofesores de la asignatura, por su constante diálogo y retroalimentación en las ideas contenidas en este escrito. Así mismo, también es invaluable el aporte de los y las estudiantes que han participado en la materia en estos años, cuya pasión por el tema y sus constantes comentarios son la energíafundamental de estas reflexiones. 


\section{Bibliografía}

Caradonna, J. (2014). Sustainability: A History. Oxford: Oxford University Press. Comité Invisible, 2015. A Nuestros Amigos. Logroño: Pepitas de Calabaza.

Díaz, F. (2007). Comunalidad, energía viva del pensamiento mixe. México: UNAM. Federici, S. (2013). Calibán y la bruja. Mujeres, cuerpo y acumulación originaria. México: Pez en el Árbol.

Federici, S. y Caffentis, G. (2015). Comunes contra y más allá del capitalismo. El Apantle, 1, 55-73.

García, A. (2009) [1995]. Forma valor y forma comunidad. Aproximación teóricoabstracta a los fundamentos civilizatorios que preceden al Ayllu universal. La Paz: Clacso/Muela del Diablo/Comuna.

Gutiérrez, R. (2001). Forma Comunal y Liberal de la Política: de la soberanía social a la irresponsabilidad civil. En grupo Comuna (Ed.), Pluriverso: Teoría política boliviana (pp. 55-73). La Paz: Muela del Diablo.

Gutiérrez, R. (2017). Horizontes comunitario-populares. Producción de lo común más allá de las políticas estado-céntricas. Madrid: Traficantes de Sueños.

Hardin, G. (1968). The Tragedy of the Commons. Science, 162, 1243-1248.

Illich, I. (2007) [1982]. El silencio es un bien comunal, Ojarasca, 117, 3-5.

Kanahuaty, C. (2015). El campo intelectual en Bolivia: el Grupo Comuna. Ecuador debate, 94, 159-170.

Locke, J. (2017) [1690]. Segundo tratado sobre el gobierno civil. Madrid: Alianza Editorial.

Linsalata, L. (2015). Cuando manda la asamblea. Lo comunitario-popular en Bolivia: una mirada desde los sistemas comunitarios de agua en Cochabamba. La Paz: SOCEE.

Martínez, J. (2010). De eso que llaman comunalidad. Oaxaca: Conaculta.

Martínez, J. (2015). Educación Comunal. Oaxaca: Casa de las Preguntas.

Marx, K. (2015) [1842]. En defensa de los ladrones de leña. En Daniel Bensaid (Ed.), Contra el expolio de nuestras vidas, una defensa del derecho a la soberanía energética, a la vivienda y a los bienes comunes (pp. 9-54.). Madrid: Errata Naturae.

Marx, K. (1998) [1861]. El Capital, México: S. XXI, pp. 221-245.

Marx, K. y Engels, F. (1978). Acerca del colonialismo (artículos y cartas). Madrid: Júcar.

Navarro, M. (2016). Hacer común contra la fragmentación en la ciudad. Experiencias de autonomía urbana. Puebla: ICSyH-BUAP.

Ostrom, E. (1990). Governing the Commons. The evolution of Institutions for Collective Action, Cambridge: Cambridge University Press.

Patzi, F. (2004) Sistema comunal. La Paz: Comunidad de Estudios Alternativos. 
Revista LIDER, 37(22), 2020, pp. 44-62

Raffo, B. (1949). Bien común y política en la concepción filosófica de Santo Tomás de Aquino. Actas del primer congreso nacional de filosofía (pp. 2022-2032). Mendoza: Universidad de Mendoza.

Salazar, H. (2015). "Se han adueñado del proceso de lucha": horizontes comunitario-populares en tensión y la reconstitución de la dominación en la Bolivia del MAS. La Paz: SOCEE/Autodeterminación.

Terrazas, J. D. (2012). Las Res Communes Omnium" Revista de Estudios HistóricoJurídicos, 34, 127-163. 